\title{
Different Methods for Determining Porosity of Gas Diffusion Layer using X-ray Microtomography
}

\author{
S. Hasanpour, M. Hoorfar, A.B. Phillion
}

School of Engineering, The University of British Columbia, Kelowna, BC V1V1V7, Canada

\begin{abstract}
Gas diffusion layer (GDL) is a crucial component in polymer electrode membrane fuel cells. Being highly porous, this layer facilitates transport of species from the flow field to the reaction sites and vice versa. One of the main characteristics of GDLs is porosity, which has been measured using a number of different methods including 3D X-ray microtomography ( $\mu$ XCT). Despite the extensive use of this technique in investigating the properties of GDLs, there are variations in the results since the surface of the three dimensional volume used to obtain the bulk porosity of GDLs is difficult to quantify. In this paper, a robust surface identification method, referred to as "Rolling Ball", is introduced to identify systematically the surface and hence porosity of GDLs from $\mu \mathrm{XCT}$ datasets. In this method, the diameter of the GDL carbon fiber is used as the characteristic length in combination with a Distance Transform (DT) to robustly identify the surface topology. This method is then used to estimate porosity of four different samples of a highly porous GDL, SGL 25BA. The results between different samples show great consistency. A comparison with other methods is also performed, and variations in the bulk and in-plane porosity are observed. The main advantage of the proposed Rolling Ball method compared to prior methods used in the literature is that it uses the carbon fiber diameter to identify the surface results in a systematic fashion. This methodology can be easily applied to other highly porous media.
\end{abstract}




\section{Introduction}

The proton exchange membrane fuel cell (PEMFC) is a promising technology that has a great potential to create electrical power and replace fossil fueled energy conversion devices. Due to its high efficiency, zero emission, and low working temperature, PEMFC is a predominant choice for the automotive industry $[1,2]$. In essence, PEMFC creates the electrical power through an electrochemical reaction during which hydrogen (i.e., fuel) is reacting with oxygen (i.e., oxidant) producing heat, water and electrical power. The PEMFC generally consists of a catalyst coated membrane $(\mathrm{CCM})$ across which the protons are transferred from the anode (where the fuel is oxidized, producing protons and electrons) to the cathode (where the protons, oxygen and electrons react, producing water). The CCM is supported on both sides by a porous gas diffusion layer (GDL) and a bipolar plate, providing passages for transport of reactants, water, and electrons to or from the CCM [3]. In some applications [3], the catalyst layer (CL) is directly deposited on the GDL, forming a gas diffusion electrode (GDE) layer supporting the solid membrane. The GDL also provides mechanical support for the membrane as well as removing heat generated during reaction $[2,4]$. The GDL has been thoroughly characterized using different methods [2], resulting in identifying important factors affecting the performance of PEMFC. One of the most important properties of the GDL is its porosity, since it provides a qualitative measure of the layer's effectiveness in transporting different species. Porosity also plays an important role in other transport properties of the GDL including permeability, diffusivity and tortuosity [5].

There are two categories of techniques used for determination of porosity of GDLs: destructive and non-destructive. Mercury Intrusion Porosimetery (MIP) [6] is recognized as the most commonly used destructive methods, while techniques like X-ray microtomography $(\mu \mathrm{XCT})$ fall 
into the non-destructive category. $\mu \mathrm{XCT}$ is an imaging technique that provides the $3 \mathrm{D}$ internal structure of material with very high resolution [7]. Sinha et al. [8] were the first group using $\mu$ XCT (with a $10-\mu \mathrm{m}$ resolution) for imaging GDLs. Their interest was to visualize the water distribution inside a GDL, which resulted in estimating an optimum purging time of water from the samples. The use of $\mu$ XCT to determine porosity in GDLs was first proposed by Buchi et al. [9], who measured porosity distributions along the thickness of GDLs. Bazylak and coworkers, in a series of comprehensive studies [7, 10-14], employed $\mu$ XCT to categorize different GDLs. First, they showed that porosity distributions of felt-based GDLs are more homogenous than those of paper-based [10]. In their next study [11], they investigated the effect of polytetrafluroethylene (PTFE) on the porosity distribution through the thickness of GDLs, showing that PTFE is distributed non-homogenously across the thickness of the GDL. They also studied the effect of cracks on the effective porosity of the micro porous layer (MPL) using $\mu$ XCT. They found that, within the MPL, the percentage porosity related to cracks steadily decreases toward catalyst layer. However, as the voxel size of the tomographic imaging was 2.44 $\mu \mathrm{m}$, smaller porosity including the pores within the MPL were not captured.[12]. In another study, Bazylak's group [13] conducted imaging of both paper and felt -based GDLs compressed to $1.2 \mathrm{MPa}$, and compared the measured porosity distributions to uncompressed GDLs. Their results showed that the effect of compression is not uniform through the thickness of samples, and the felt -based GDL is more sensitive to compression in comparison with paper-based GDLs. James et al. [14] also examined the effects of compression on the transport properties of GDLs by creating a sample holder mimicking the channels and ribs of the bipolar plate. Using $\mu$ XCT for imaging the resulting structure, they showed that the area under compression has significantly less porosity. By developing a computational fluid dynamics (CFD) model based on the $\mu$ XCT- 
derived geometry they found that the transport properties in the region under compression differ by a factor of 2 in comparison with the un-compressed regions.

The analysis of $\mu \mathrm{XCT}$ datasets to observe the 3D structure of materials involves several steps, including filtering, binarization and volume rendering. Among them, the most challenging step is binarization, which is used to differentiate the material from background. This step requires a threshold value. The most common method for thresholding is Otsu's method [15], which has been used to analysis different GDLs [10,14]. In one study, Ostadi et al. [16] investigated the effect of different threshold values on the properties of the GDLs. This was accomplished by first visually selecting a global threshold value, and then varying the threshold value in increments of 1 grayscale units, calculating porosity at each increment. The results indicated that porosity changes by $2 \%$ by changing the threshold value by $3 \%$. Following this, Ostadi et al. [17] compared the 3D images of GDL obtained using $\mu$ XCT with the SEM images. The fiber diameter obtained from the SEM image was used to identify the right threshold value for processing of $\mu$ XCT images. Odaya et al. [18] used a global thresholding method (instead of the Otsu method [15]) to extract the GDL phase from the background, and compared porosity in single and dual-layer GDLs. Although global thresholding is considered unsophisticated, the results showed that for some cases the use of a global threshold is equivalent to and sometimes even better than those obtained from the Otsu method [15].

Regardless of the image processing steps employed for determining porosity of the sample, the identification of the surface of the sample remains an open question. Assuming that porosity is calculated by $\varepsilon=\left(1-V_{\mathrm{GDL}}\right) / V_{\text {total }}$, where $V_{\mathrm{GDL}}$ is the volume of the GDL material and $V_{\text {total }}$ is the total volume, surface identification is key since it affects $V_{\text {total }}$. The problem in highly 
porous materials with porosity more than $80 \%$, like GDLs, is that the surface is not clear and/or quite rough. In most studies mentioned above, subjective assumptions were made to identify the surface of the GDL. Fishman et al. [10] assumed the surface of GDL to be where the GDL fiber is present in a $2 \mathrm{D}$ image in amounts greater than $1 \%$. This method, which is referred to as the 99\% Porosity method here, requires that the 3D $\mu \mathrm{XCT}$ dataset be perfectly aligned with the global coordinate system. Ostadi et al. [16] assumed a cubic bounding box to measure GDL porosity, resulting in porosity being a qualitative measure only - dependent on the bounding box size. Another method is to take the mean surface height, which is the standard definition for quantifying surface roughness of bulk materials. In this study, a robust method inspired from paper physics [19], which we name the Rolling Ball method, is proposed to define the surface of GDLs and consequently the GDL bulk porosity. Unlike the previously proposed arbitrary methods, the proposed Rolling Ball method uses the actual dimensions of the fibers to identify the surface. Thus, it provides a systematic method of determining the porosity of GDLs and comparing between materials and manufacturing methods.

\section{Methodology}

In this section, the description of the GDL material is provided along with the procedures used to acquire the $\mu \mathrm{XCT}$ images and to binarize the resulting 3D datasets.

\subsection{Materials}

One sheet of the GDL material SGL25BA was used for this investigation. SGL25BA is a single layer GDL (with no MPL) with $5 \mathrm{wt} \%$ PTFE loading and specified thickness of $190 \mu \mathrm{m}$ [20]. Four samples, from different parts of the sheet, were used in order to show consistency of the methods and results. 


\subsection{Scanning procedure}

The tomographic imaging was performed using a Zeiss $\mu \mathrm{XCT}-400$ computed tomography microscope following the procedure outlined in Odaya et al. [18]. Briefly, the SGL25BA samples were cut to a size of $4 \mathrm{~mm}^{2}$ in cross-section and firmly secured to the sample holder to minimize sample flutter during image acquisition. Approximately an area of $1 \mathrm{~mm}^{2}$ was imaged at a voxel size of $1.167 \mathrm{~mm}$, resulting in a volume consisting of approximately $900 \times 900 \times 200$ voxels. This size of the sample is large enough for investigating the porosity distribution [10].

\subsection{Image processing}

Each 3D data set was post-processed to reduce noise and to convert to the binary form (black and white) using the ImageJ, MATLAB and Avizo software packages. First, a median filter was applied on the images to reduce noise. Since the purpose of this study is to identify the GDL surface, no effort was made to separate the binder/PTFE and carbon fiber. Second, a manual threshold value $[18,20]$ was applied to segment the GDL from the background. As approximately $5 \%$ of the volume (at the top and bottom) was quite noisy, it was cropped from the dataset.

\section{Porosity quantification}

\subsection{Rolling Ball method}

Figure 1-a shows the binary image of a cross section of a 2D image. The black (white) part represents void (GDL material). Figure 1-b shows the corresponding 3D view of all the 2D images stacked on top of each other. As it can be seen, the carbon fiber strands are randomly distributed. This intrinsic feature of the GDL makes porosity highly variable from one position to 
another. Some parts consist of very large pore sizes and other areas have very small pores. The high surface roughness present on GDLs can be clearly seen in Figure 1, especially in the cross sectional view. In order to accurately and robustly quantify porosity in GDLs, the surface of this highly porous material must be identified.

The Rolling Ball method for porosity determination uses a well-known image processing technique known as Rolling Ball [19, 20, and 22] for surface identification that is based on distance transforms. The Rolling Ball technique is so-named because it can be thought of as the path taken by a ball as it moves over a surface in 3D. The radius of the ball relative to the true surface roughness defines the identified surface. The developed method is outlined in Figure 2 on a 2D example set of images, although the method was applied directly to the 3D dataset. First, the image is binarized (Figure 2-a). Second, a Euclidean distance transform function is applied (Figure 2-b). This function measures the distance between each voxel from the closest white voxel, and then the value for this distance is stored in that same voxel. Third, a value for $R$, representing the rolling ball radius, is chosen to identify the surface. Each voxel that has a value higher than $R$ is labeled black, while values less than or equal to $R$ are labeled as white. Figure 2$\mathrm{c}$ shows the output for $R=15$ voxels. Finally, through binary multiplication of Figure 2-a and 2-c, the GDL surface and the internal pores are fully revealed.

With a well-defined surface, porosity can be estimated on a line-by-line basis going through the plane of the GDL. Figure 3 shows the original binarized image on the left, and the combined rolling ball area and the GDL material on the right. A grey line has been added from the top to the bottom surface to demonstrate the calculation of line-by-line porosity. The porosity along each line is calculated by dividing the white voxels by the total number of voxels along the line. 
By repeating this measurement throughout the $3 \mathrm{D}$ dataset, the $3 \mathrm{D}$ porosity for whole volume is calculated.

The most important parameter in the above algorithm is the choice of the rolling ball radius. Figure 4 shows the effect of the $R$ value on the identified surface. As it can be seen, by increasing $R$, the surface moves outward, increasing the area identified as "inside" the GDL. Thus, increasing $R$ leads to an increase in apparent GDL porosity. Figure 5 shows the variation in bulk porosity for SGL 25BA as a function of $R$. The difference in porosity with the rolling ball radius is considerable, ranging from 0.65 to 0.90 for the same sample.

In order to define GDL porosity in a systematic fashion, the rolling ball radius should be defined as a function of another intrinsic property. One intrinsic property of paper-based GDLs that is readily available is the radius of the carbon fibers. All paper-based GDLs are fabricated with fibers of a known and specified radius, which is not changed by PTFE loading. Using the fiber diameter, the selection of the rolling ball radius can thus be directly linked to the characteristics of the GDL, enabling robust porosity estimation between different GDLs.

\subsection{Alternative methods}

99\% Porosity: In addition to determining the bulk porosity, the $\mu$ XCT datasets of GDLs can be used to calculate the porosity variation along each direction of the sheet. Fishman et al. [10] employed this knowledge to plot porosity distributions along different axes. Along the thickness of the GDL, the "through-plane" porosity will approach $100 \%$ as the surface of the GDL is reached. Fishman et al. [10] assumed that the bulk porosity was defined using a bounding box that was large enough to encompass all GDL material until the through-plane porosity reached a value of $99 \%$. 
Mean Surface Height: Although the surface itself is difficult to identify in Figure 1, the mean surface position, $\bar{Y}$, for the top of the sheet can easily be calculated (using an arbitrary coordinate

system) by $\bar{Y}=\frac{\sum y}{n}$, where $y$ is the position of the first white particle in the 2D image and $n$ is the number of columns in the 2D image. A similar procedure can be used to calculate the bottom surface.

Figure 6 shows a 2D cross-sectional image, with white voxels representing the GDL material and black voxels representing voids. The solid and dashed lines represent the top and bottom surfaces identified by the $99 \%$ Porosity and Mean Surface Height methods, respectively, which there is considerable difference in the position. The $99 \%$ Porosity method creates a very large total volume for porosity estimation, while the Mean Surface Height method only covers a very small portion of the volume. The resulting bulk porosity estimates will vary considerably, and, especially in the case of the $99 \%$ Porosity method, be strongly linked with manufacturing variations.

\section{Results and Discussion}

Figure 7 shows two scanning electron microscopy images, each taken at a different magnification, which were used to estimate the radius of the carbon fiber in SGL25BA. A series of fiber cross-sections were measured at both resolutions and then averaged. The use of two images at two different resolutions resolves the variation in the fiber radius while allowing for a sufficient number of fibers to be measured. Using this technique, a fiber radius of $6.5 \mu \mathrm{m}$ has been determined for this specific GDL.

Bulk porosity estimated using the Rolling Ball, 99\% Porosity, and Mean Surface Height methods is summarized in Table 1 for all four samples of SGL25BA scanned using $\mu$ XCT. The $99 \%$ 
Porosity method gives the highest values (avg. 87\% porosity), and the Rolling Ball method gives the lowest values (avg. 77\% porosity). Since the underlying assumptions for the total volume of the bounding box are different, the values are expected to be different. Furthermore, there are also some differences in the porosity estimation between different samples, with the Rolling Ball method providing the largest variation, between $75 \%$ and $79 \%$. The main advantage in using the Rolling Ball method is that it is a robust measure of porosity, enabling comparisons between GDLs without arbitrary definitions of the surface. Thus, the variation in porosity between the four samples proposed by the Rolling Ball method truly exists, and represents the variation in internal porosity, and not just differences in the size of the bounding box.

The porosity distribution along different axes is a key parameter in porous media, and is often reported for GDL materials. The variation in the in-plane porosity calculated using all three porosity estimation methods is shown in Figure 8. As it can be seen, different assumptions leads to different values of porosity; the porosity based on the $99 \%$ Porosity method is significantly higher throughout the GDL as compared to the Rolling Ball and Mean Surface Height methods, similar to values listed in Table 1. The standard deviation of the in-plane porosity was also calculated. The $99 \%$ Porosity method gives a smooth result (S.D. $=0.020)$, while the other two methods have more significant variations. This result was expected given that the Rolling Ball method (S.D. $=0.038)$ and to some degree the Mean Surface Height method (S.D. $=0.040)$ are able to capture all of the variations in surface topography. Finally, Figure 9 shows the variation in the through-plane porosity for the four samples of SGL25BA, calculated using the Rolling Ball method. Although the general trends are the same, there are some variations in porosity between different regions of a single GDL sheet. 
Among all of these methods, only the Rolling Ball method is able to generate a porosity estimation based on the actual properties of the GDL material. This allows for comparison of different GDLs in a systematic fashion for subsequent determination of permeability, tortuosity, and diffusivity based on analytical expressions. Furthermore, it is extremely important that the research community agree on a robust and straightforward porosity estimation method. Otherwise, the reported value is less useful since with different assumptions, the values will change significantly. To give an example, the permeability calculated for the $1^{\text {st }}$ sample using the Tomadakis and Sotirchos analytical expression [5] is $13.1 \times \mu \mathrm{m}^{2}$ based on the Rolling Ball method and $67.5 \times \mu \mathrm{m}^{2}$ for the $99 \%$ Porosity method.

\section{Conclusions}

In this study, $\mu$ XCT has been used to obtain the 3D structure of GDLs at a high resolution of approximately $1 \mu \mathrm{m}$ voxel size. This $3 \mathrm{D}$ data provides fine details about the internal structure of GDLs, which can be used for property estimation. The crucial parameter of a GDL is porosity, since this is the main microstructural property governing the transport properties (permeability, diffusivity, and tortuosity) of the material. The Rolling Ball method has been introduced to systematically measure the GDL porosity. This method uses the intrinsic property of the material, fiber diameter, to identify efficiently the fiber surface and hence estimate porosity. In comparison to previous methods, like the $99 \%$ Porosity and Mean Surface Height methods, the Rolling Ball method takes into account the complex topology and underlying structure.

\section{Acknowledgements}


The authors thank the Natural Sciences and Engineering Council of Canada for funding this research, and the Canada Foundation for Innovation, for funding the purchase of the X-ray tomographic microscope.

\section{References}

[1] B. Frano, PEM fuel cells: theory and practice, (2005).

[2] A. Arvay, E. Yli-Rantala, C. Liu, X. Peng, P. Koski, L. Cindrella, et al., Characterization techniques for gas diffusion layers for proton exchange membrane fuel cells-A review, J. Power Sources 213 (2012) 317-337.

[3] C. Liu, C. Sung, A review of the performance and analysis of proton exchange membrane fuel cell membrane electrode assemblies, J. Power Sources 220 (2012) 348-353.

[4] M. Mathias, J. Roth, J. Fleming, W. Lehnert, Diffusion media materials and characterisation, Handbook of fuel cells (2003).

[5] M.M. Tomadakis, T.J. Robertson, Viscous permeability of random fiber structures: comparison of electrical and diffusional estimates with experimental and analytical results, J. Composite Mater. 39 (2005) 163-188.

[6] R.R. Rashapov, J. Unno, J.T. Gostick, Characterization of PEMFC Gas Diffusion Layer Porosity, J. Electrochem. Soc. 162 (2015) F603-F612.

[7] P.R. Challa, X-ray Investigations of PEMFC Gas Diffusion Layers (GDLs) (2012).

[8] P.K. Sinha, P. Halleck, C. Wang, Quantification of liquid water saturation in a PEM fuel cell diffusion medium using X-ray microtomography, Electrochemical and Solid-State Letters 9 (2006) A344-A348.

[9] F.N. Büchi, R. Flückiger, D. Tehlar, F. Marone, M. Stampanoni, Determination of liquid water distribution in porous transport layers, ECS Transactions 16 (2008) 587-592.

[10] Z. Fishman, J. Hinebaugh, A. Bazylak, Microscale tomography investigations of heterogeneous porosity distributions of PEMFC GDLs, J. Electrochem. Soc. 157 (2010) B1643B1650.

[11] Z. Fishman, A. Bazylak, Heterogeneous through-plane porosity distributions for treated PEMFC GDLs I. PTFE effect, J. Electrochem. Soc. 158 (2011) B841-B845.

[12] Z. Fishman, A. Bazylak, Heterogeneous through-plane porosity distributions for treated PEMFC GDLs. II. Effect of MPL cracks, J. Electrochem. Soc. 158 (2011) B846-B851.

[13] P.R. Challa, A. Bazylak, Pore space characterization of compressed PEMFC GDLs using 3D micro-computed tomography, (2012) 189-194. 
[14] J. James, H. Choi, J. Pharoah, X-ray computed tomography reconstruction and analysis of polymer electrolyte membrane fuel cell porous transport layers, Int J Hydrogen Energy 37 (2012) 18216-18230.

[15] N. Otsu, A threshold selection method from gray-level histograms, Automatica 11 (1975) 23-27.

[16] H. Ostadi, P. Rama, Y. Liu, R. Chen, X. Zhang, K. Jiang, Influence of threshold variation on determining the properties of a polymer electrolyte fuel cell gas diffusion layer in X-ray nanotomography, Chemical Engineering Science 65 (2010) 2213-2217.

[17] H. Ostadi, K. Jiang, P. Prewett, Micro/nano X-ray tomography reconstruction fine-tuning using scanning electron microscope images, Micro \& Nano Letters, IET 3 (2008) 106-109.

[18] S. Odaya, R. Phillips, Y. Sharma, J. Bellerive, A. Phillion, M. Hoorfar, X-ray Tomographic Analysis of Porosity Distributions in Gas Diffusion Layers of Proton Exchange Membrane Fuel Cells, Electrochim. Acta (2014).

[19] M. Aronsson, On 3D fibre measurements of digitized paper, 2002.

[20] S. Hasanpour, A. Phillion, M. Hoorfar, Investigation of Gas Diffusion Layer Properties Using X-ray Microtomography, (2015) [in press].

[21] Sigracet GDL $24 \& 25$ series gas diffusion layer, the GDL series specification. Available from: http://www.ion-power.com/res/Sigracet/GDL_24_25_Series_07.pdf

[22] F. Petra. A method to characterize the material distribution in paper, 2000. 


\section{Figure Captions}

Figure 1(a) Cross-sectional view of binarized GDL (b) 3D image of GDL $5 \mathrm{wt} \%$ PTFE (SGL25BA)

Figure 2 The processes for the Rolling Ball method: (a) cross sectional view of binarized GDL (SGL25BA); (b) Euclidean DT applied to (a); (c) the area defined as material by the Rolling Ball method.

Figure 3 The algorithm for finding porosity. The left side represents the binary image, while the right side shows the area identified by the Rolling Ball method. The linear porosity is given by dividing all the pixels along the dotted line by the internal grey pixels. The calculation can be summed in $3 \mathrm{D}$ to calculate percentage porosity.

Figure 4 Effect of the rolling ball on segmented area for (a) $R=5$; (b) $R=20$; (c) $R=45$

Figure 5 Variation in fractional bulk porosity as a function of the rolling ball radius

Figure 6 Cross sectional image of GDL, SGL 25BA, the rigid line represents the $99 \%$ Porosity method for identifying the surface, while the dashed line represents the Mean Surface Height method.

Figure 7 SEM images of SGL 25BA at (a) 200 X magnification; (b) 1000 magnification

Figure 8 The effect of rolling ball radius on the in-plane porosity

Figure 9 Variation in area porosity through the thickness of SGL25BA; the results for all 4 specimens are provided. 
7. Tables

Table 1 Porosity value based on different methods

\begin{tabular}{|l|l|l|l|}
\hline SGL 25BA & Rolling Ball $(R=6.5)$ & $99 \%$ Porosity & $\begin{array}{l}\text { Mean Surface } \\
\text { Height }\end{array}$ \\
\hline $1^{\text {st }}$ sample & 77.21 & 87.78 & 81 \\
\hline $2^{\text {nd }}$ sample & 79.27 & 87.97 & 82.87 \\
\hline $3^{\text {rd }}$ sample & 75.51 & 85.37 & 79.85 \\
\hline $4^{\text {th }}$ sample & 76.76 & 86.51 & 79.55 \\
\hline
\end{tabular}



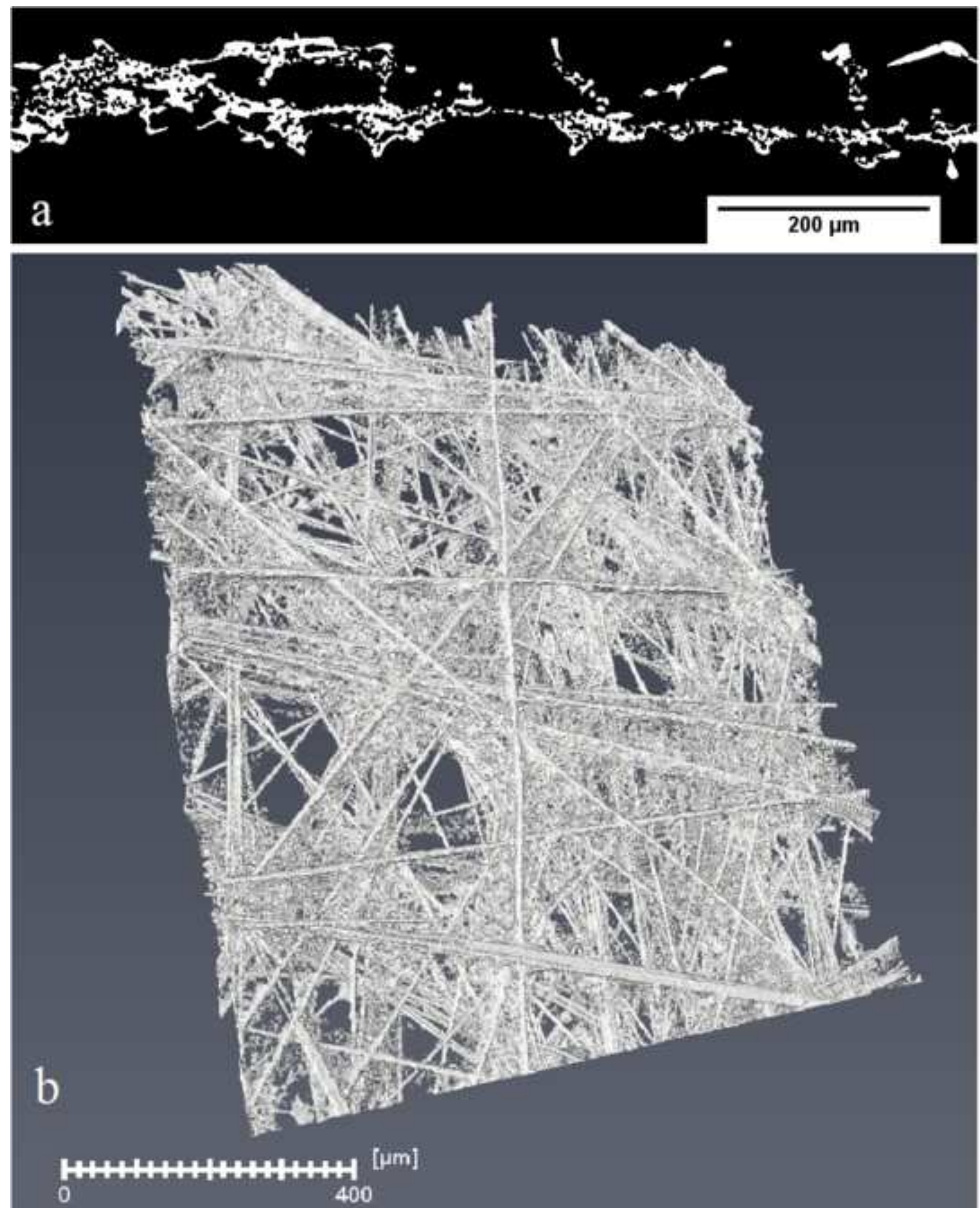
Click here to download high resolution image

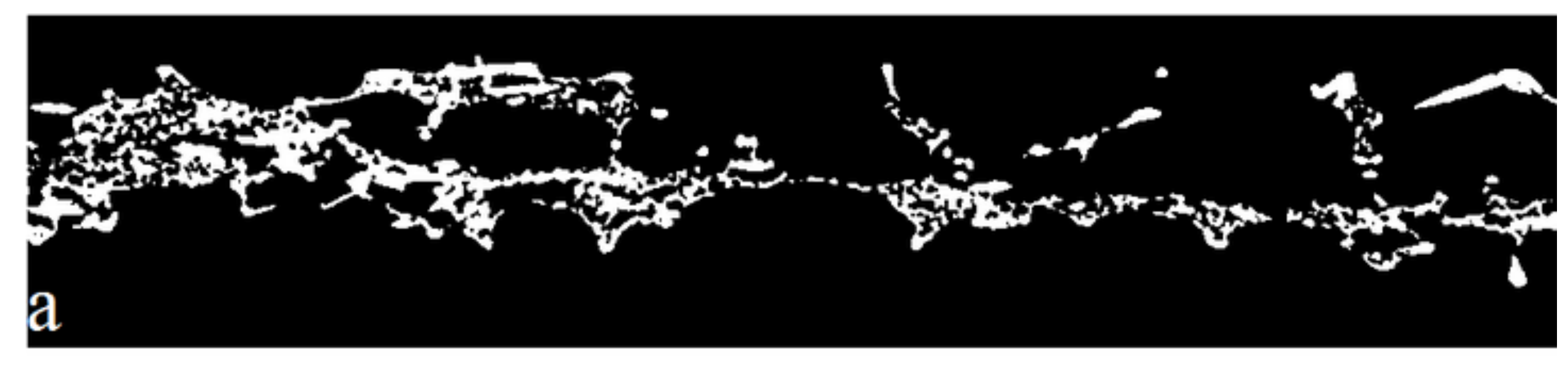

b
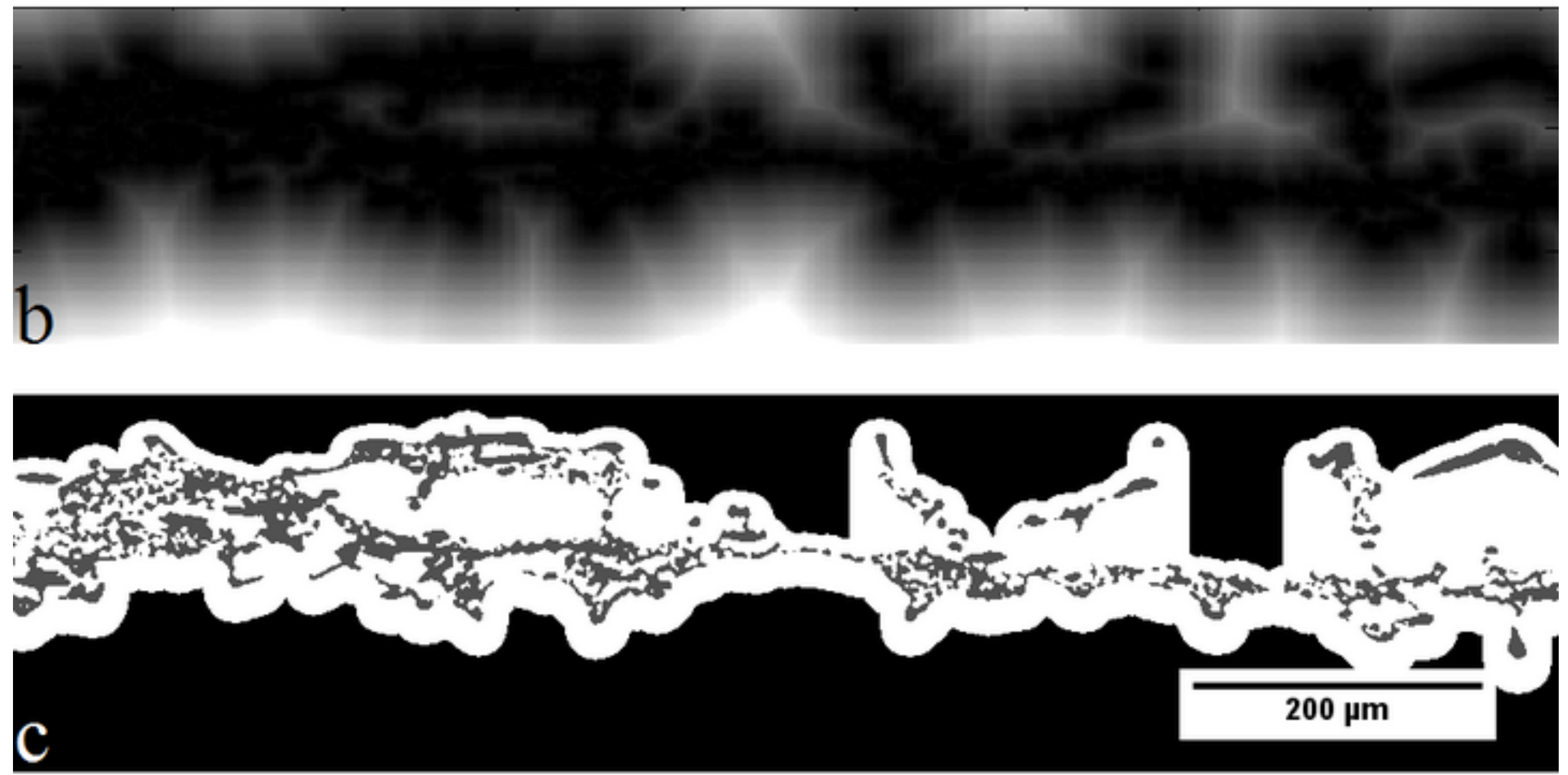


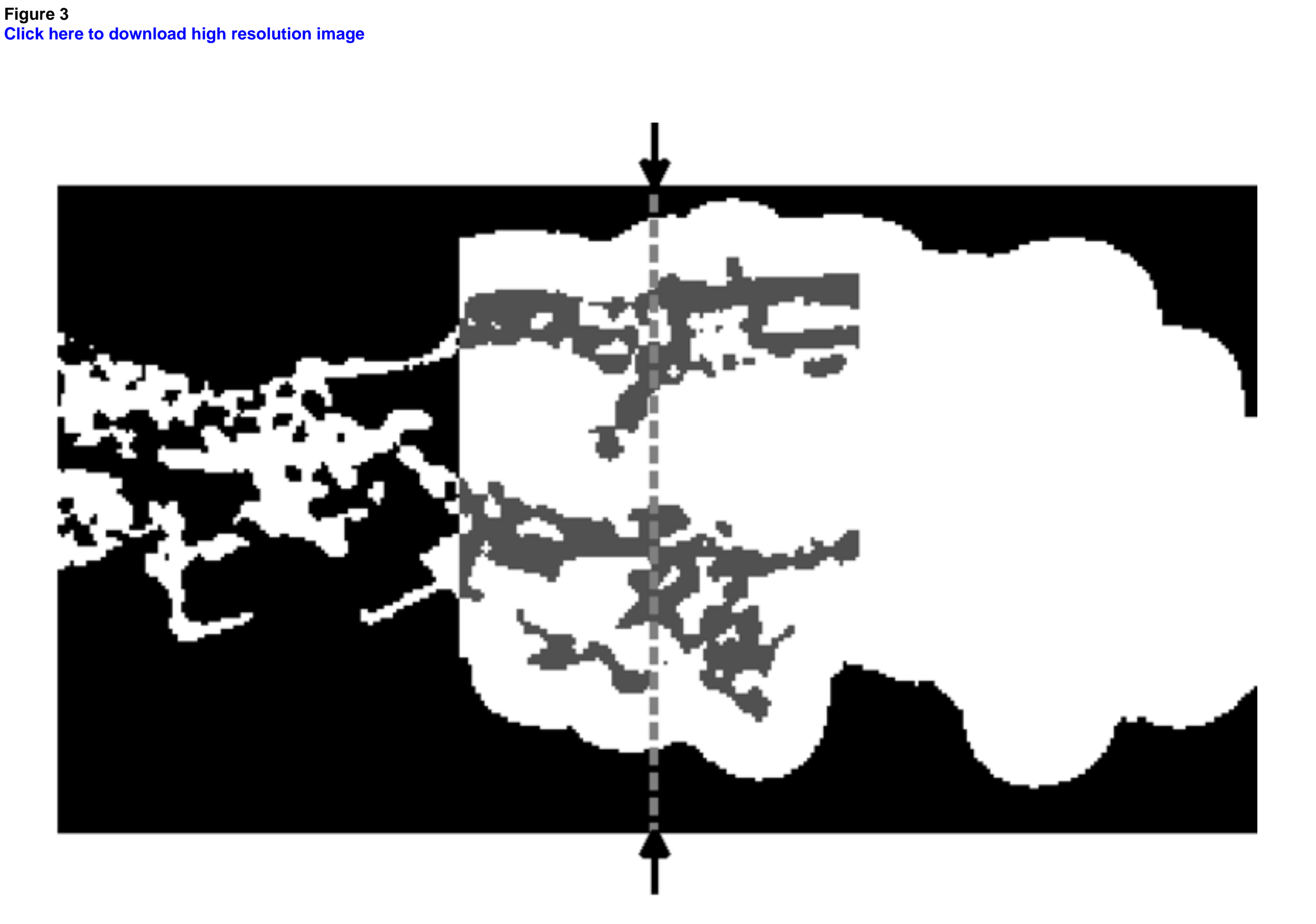

(1)

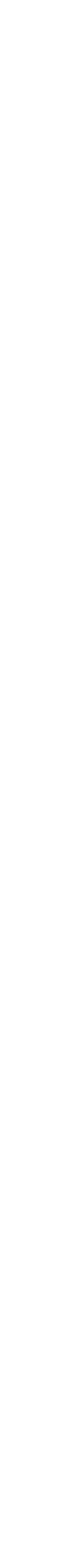


Click here to download high resolution image
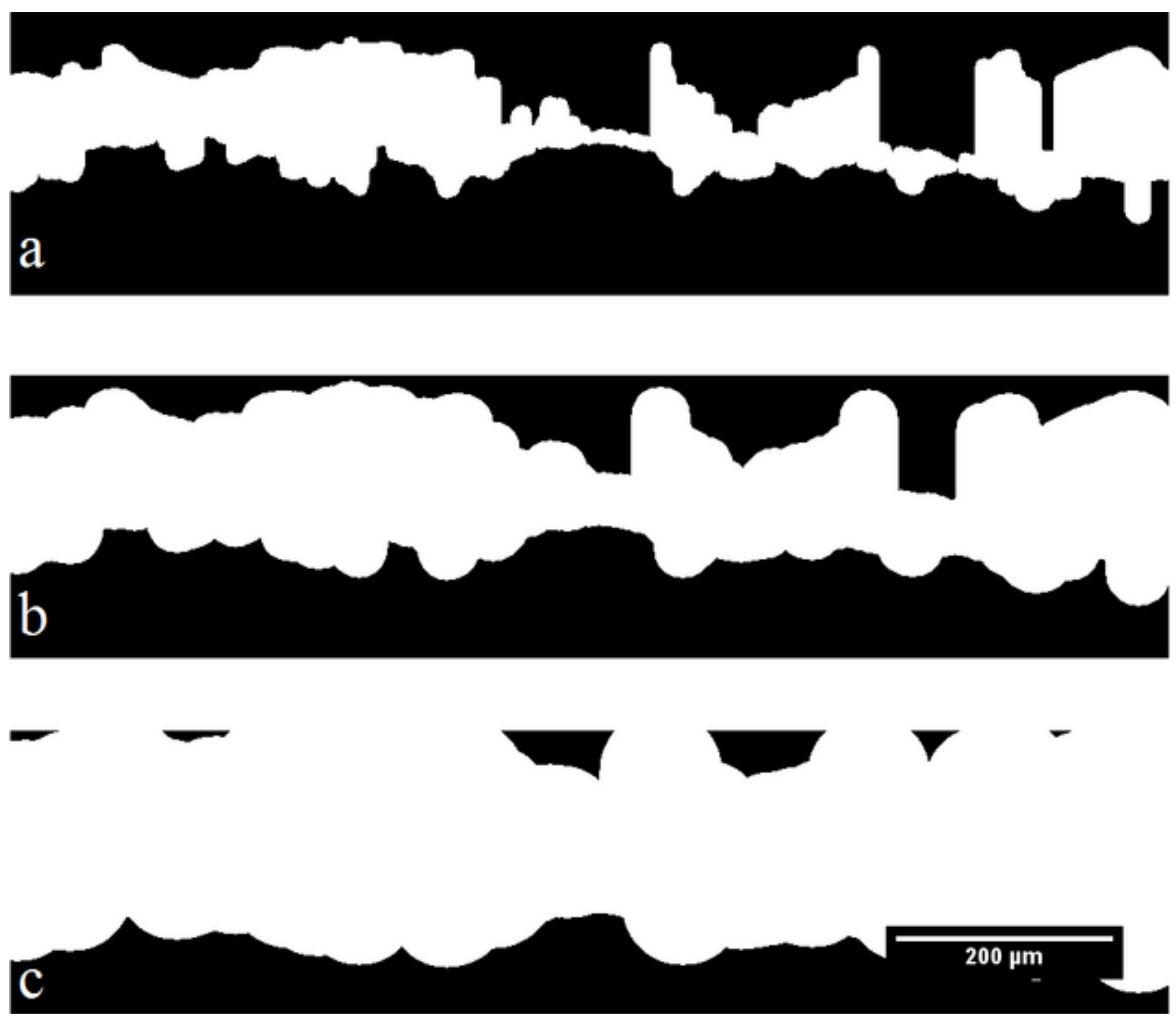
Click here to download high resolution image

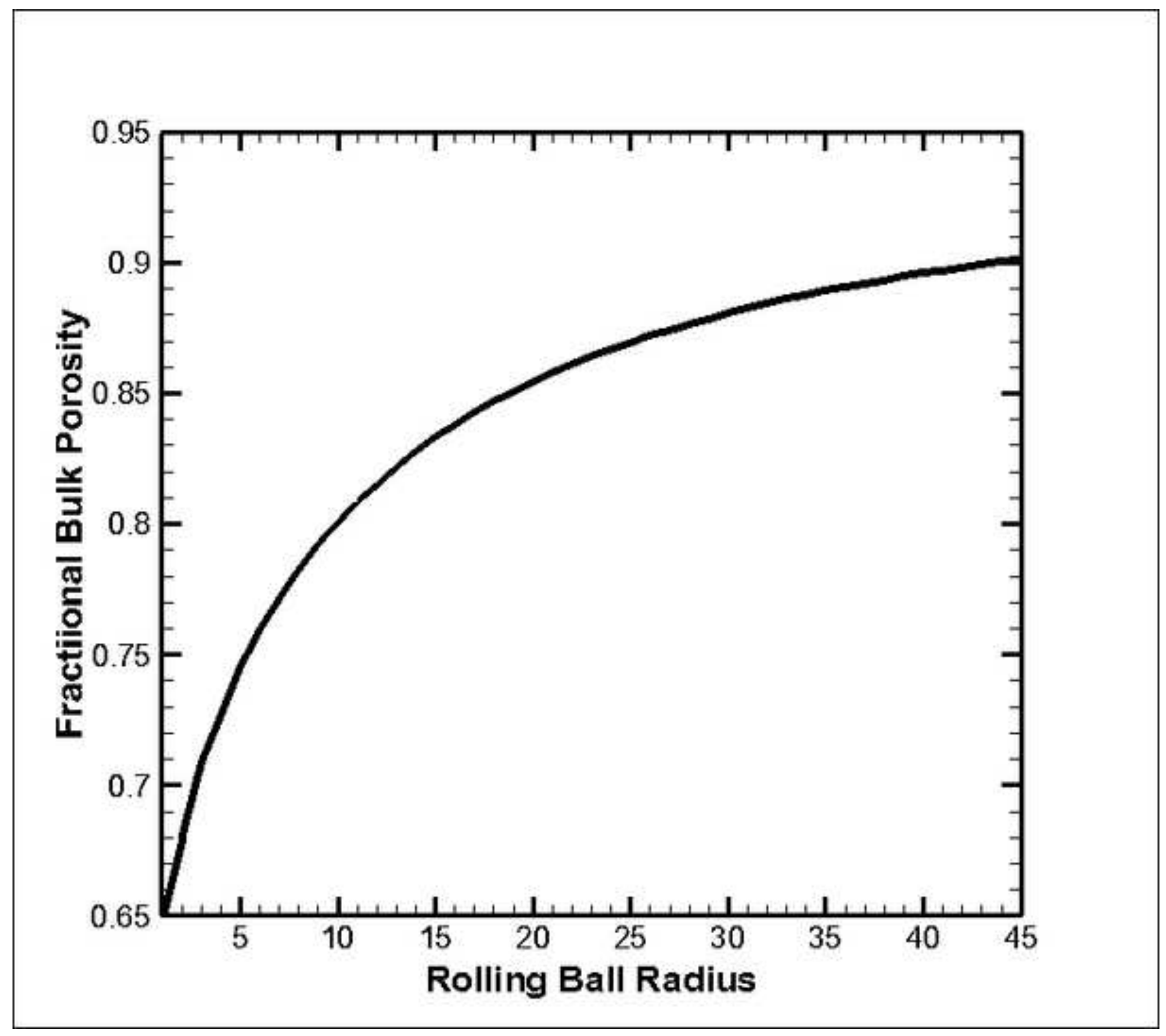


Figure 7

Click here to download high resolution image
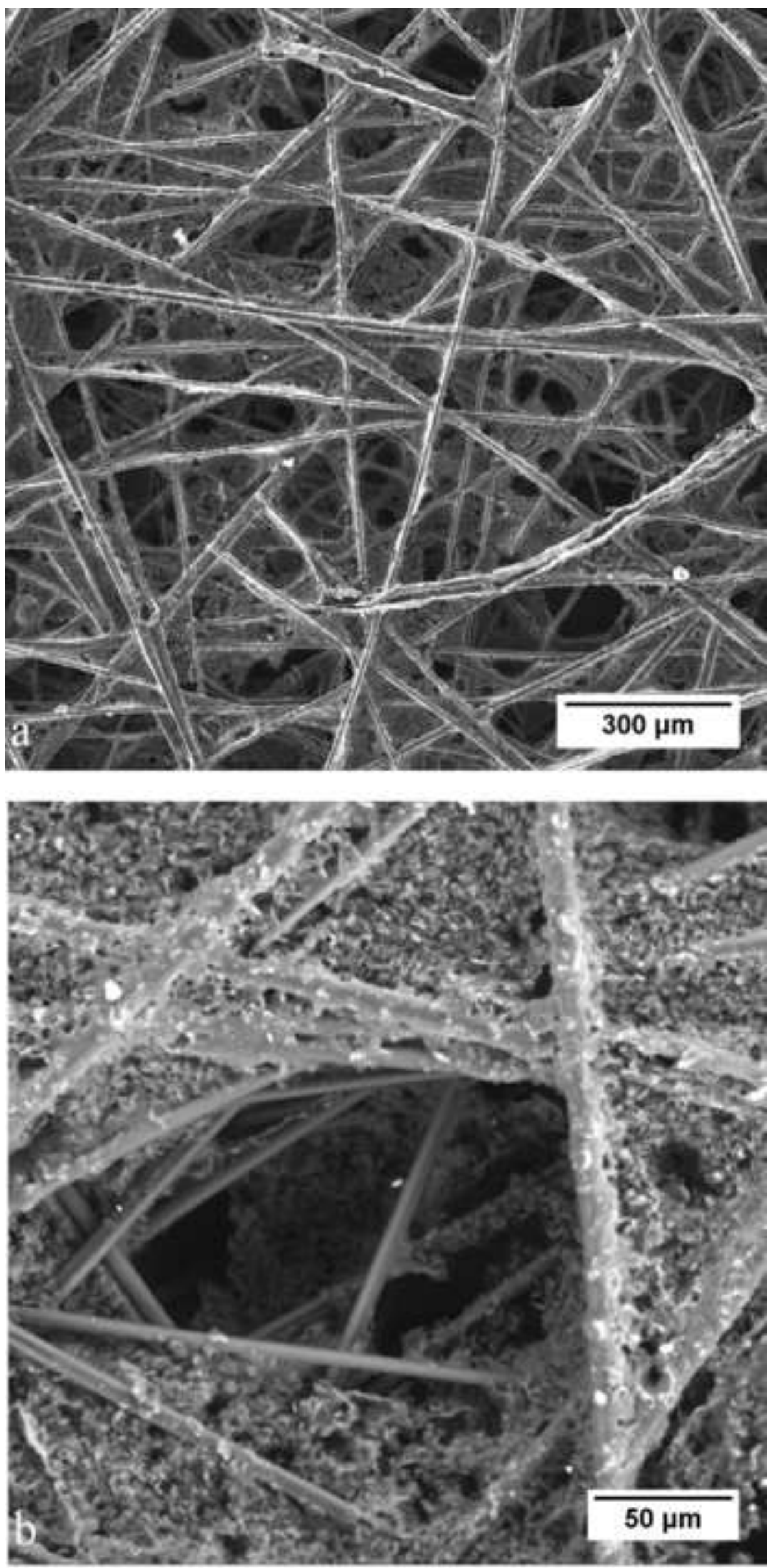


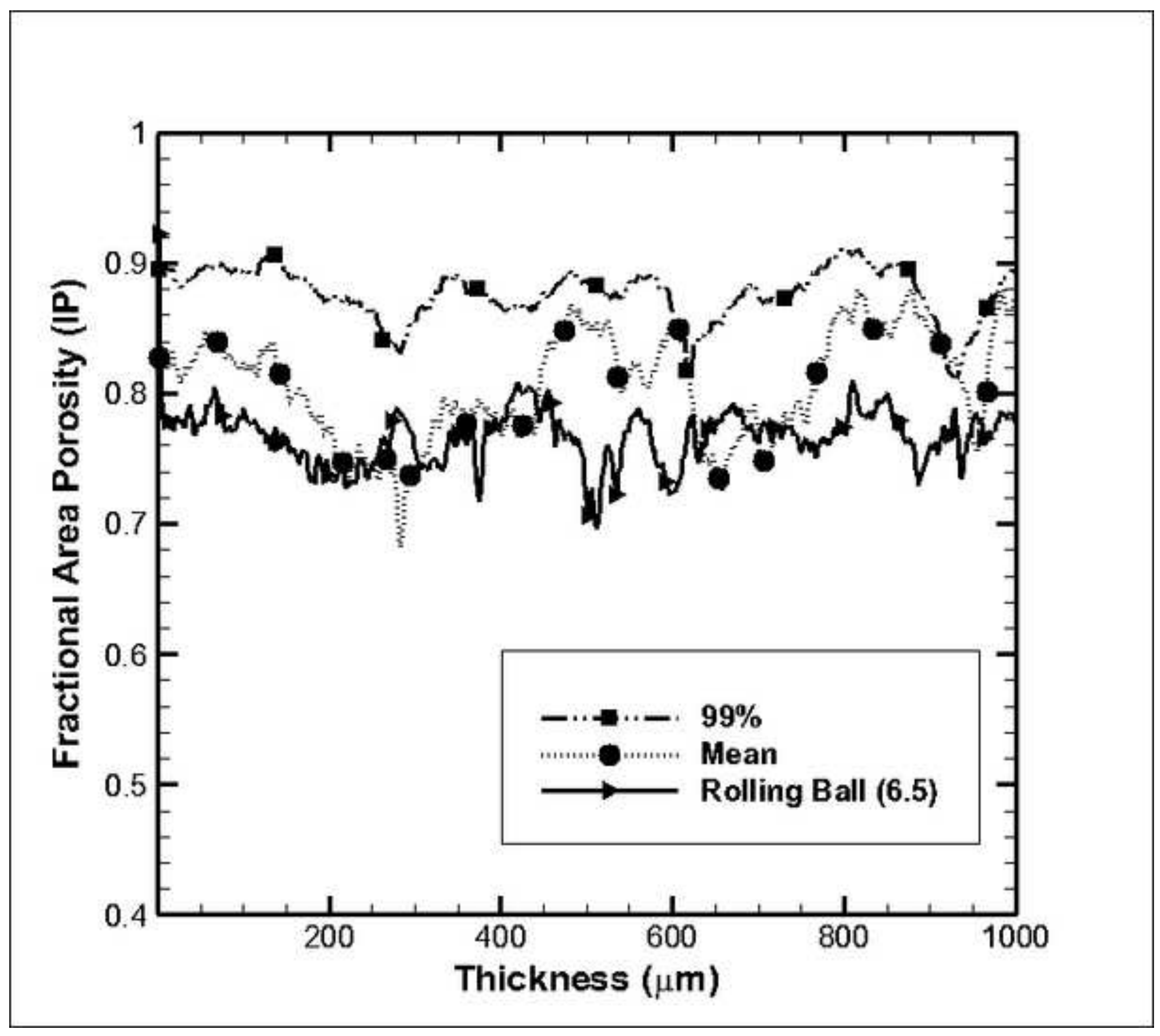




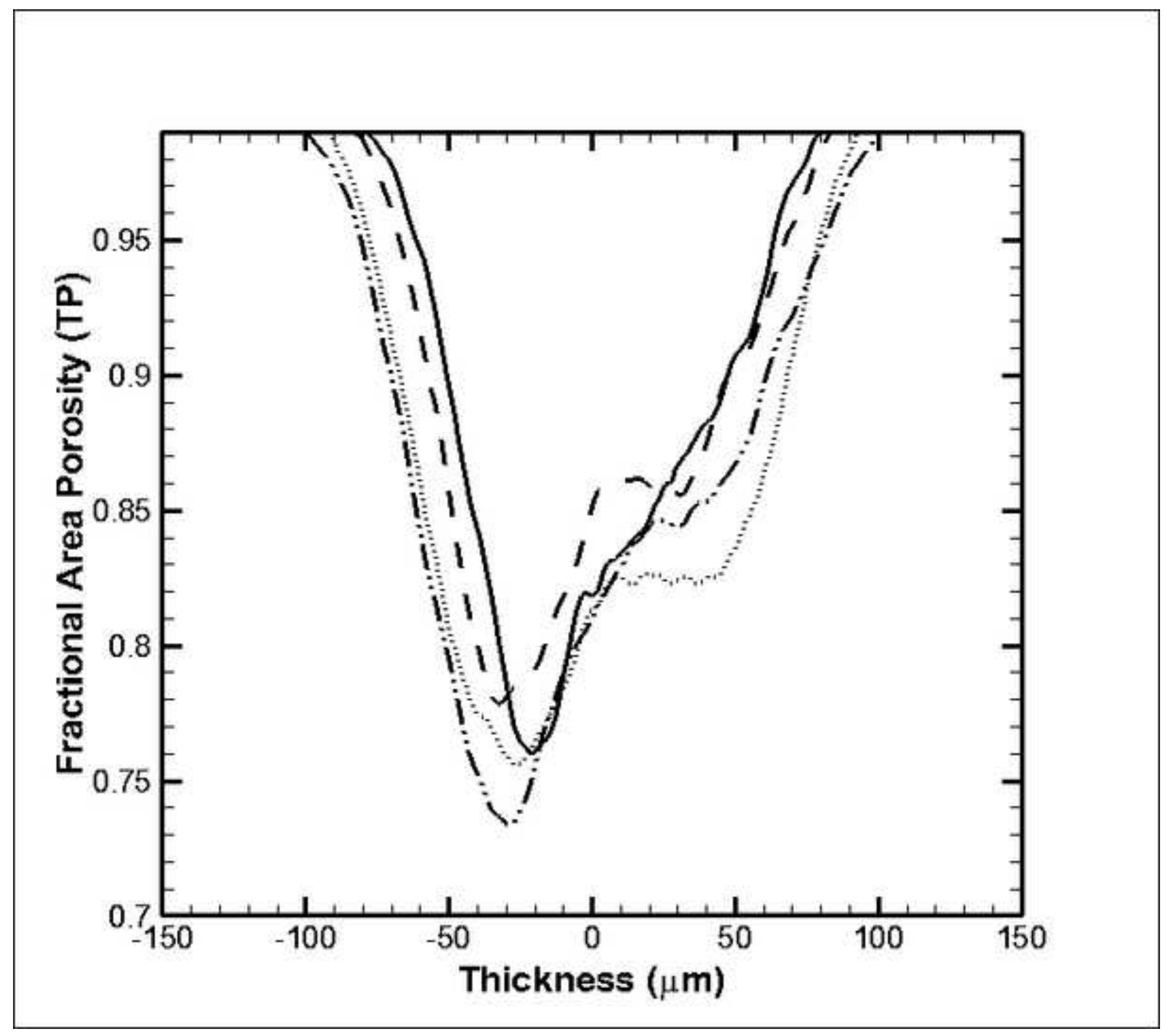

